\title{
ATM and ataxia telangiectasia
}

\section{Second in Molecular M edicine Review Series}

\author{
Peter J. McKinnon \\ St JudeChildren's Research H ospital, M emphis, Tennessee, USA
}

\begin{abstract}
Ataxia telangiectasia (AT) has long intrigued the biomedical research community owing to the spectrum of defects that are characteristic of the disease, including neurodegeneration, immune dysfunction, radiosensitivity and cancer predisposition. Following the identification of mutations in ATM (ataxia telangiectasia, mutated) as the underlying cause of the disease, biochemical analysis of this protein kinase has shown that it is a crucial nexus for the cellular response to DNA double-stranded breaks. Many ATM kinase substrates are important players in the cellular responses that prevent cancer. Accordingly, AT is a disease that results from defects in the response to specific types of DNA damage. Thus, although it is a rare neurodegenerative disease, understanding the biology of AT will lead to a greater understanding of the fundamental processes that underpin cancer and neurodegeneration.
\end{abstract}

Keywords: ataxia telangiectasia; ATM; DNA damage; neurodegeneration

EM BO reports (2004) 5, 772-776. doi:10.1038/sj.embor.7400210

\section{Introduction}

Ataxia telangiectasia (AT) is a neurodegenerative disease that occurs early in childhood (Gatti et al, 2001; Sedgwick \& Boder, 1991). Clinically, AT presents with uncoordinated or ataxic movements that are often associated with ocular telangiectasia (dilated blood vessels of the eye). O ne certain outcome of AT is that an individual will be wheelchair-bound early in life, almost always before the adolescent years. The prominent neurological sign of AT is an inexorable loss of cerebellar function, and progressive dysarthria (speech defects) and choreoathetosis (abnormal body movements; Crawford, 1998; Gatti et al, 2001; Sedgwick \& Boder, 1991). Autopsies and magnetic resonance imaging (MRI) studies have revealed significant thinning of the molecular layer of the cerebellum and cerebellar atrophy, especially in vermal regions (Farina et al, 1994; Tavani et al, 2003). Characteristic eye movement abnormalities (distinct from telangiectasia) also feature strongly in AT, and these might be related to cerebellar dysfunction (Lewis et al, 1999). Although

Department of Genetics and Tumor Cell Biology, St JudeChildren's Research H ospital,

332 N. Lauderdale, M emphis, Tennessee 38105, USA

Tel: +1 901495 2700; Fax: +1901 5262907 ;

E-mail: peter.mckinnon@stjude.org

Submitted 21 May 2004; accepted 23 June 2004 substantial advances have recently been made in the clinical diagnosis of this disease, treatments for the progressive neurodegeneration are lacking (Perlman et al, 2003).

In addition to the hallmark neurodegeneration, there are a number of other features that typify this debilitating disease. These include immune dysfunction, sterility, radiosensitivity and lymphoid cancer (Fig 1). The immunodeficiency phenotype in AT is variable

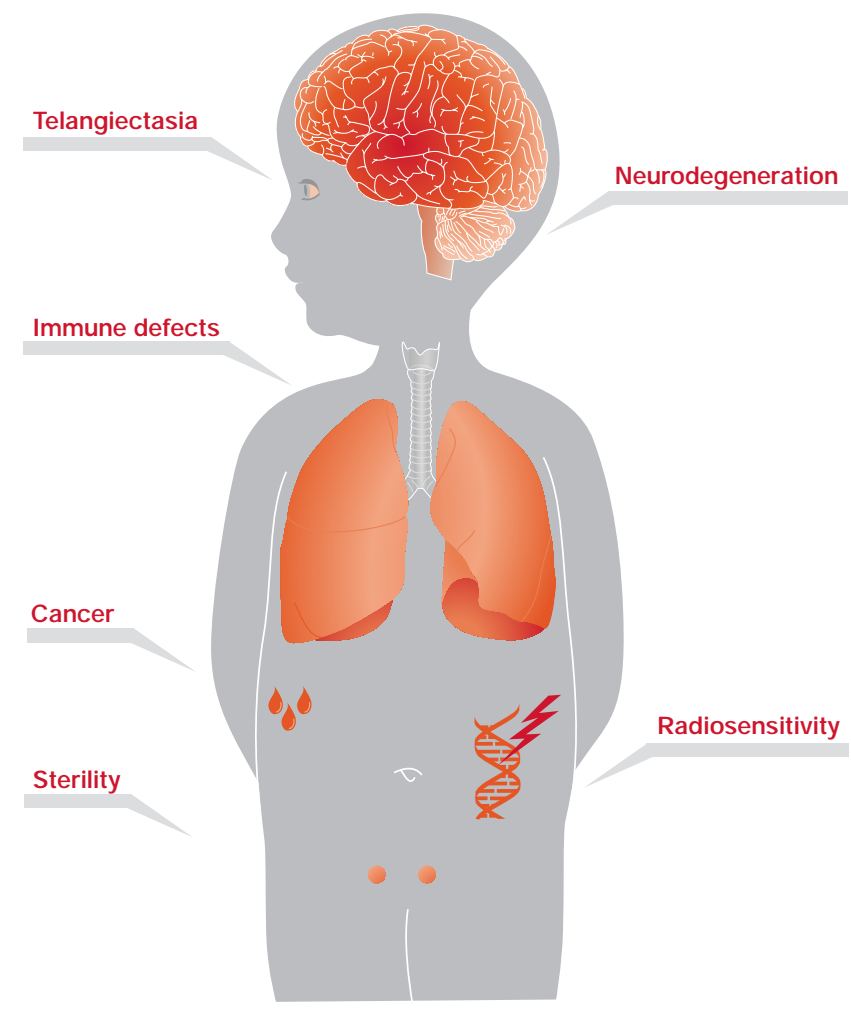

Fig1|Ataxia telangiectasia. Ataxia telangiectasia (AT) is a multisystem syndromethat results from the mutation of ATM (ataxia telangiectasia, mutated); the hallmark of clinical presentation is a debilitating progressive neurodegeneration. Other characteristics are extremeradiosensitivity, immunodeficiency, a predisposition to cancer (haematopoietic malignancy) and sterility dueto defective meiotic recombination. Ocular and facial telangiectasia arealso associated with AT . 
and usually manifests as decreased or absent IgA, IgE and IgG2, although severe bacterial or viral opportunistic infections are rare (Nowak-Wegrzyn et al, 2004). The cause of death in AT is often pneumonia or chronic lung disease, which might result from defects in chewing and swallowing owing to progressive neurological impairment (Lefton-Greif et al, 2000; N owak-W egrzyn et al, 2004). Cancer predisposition is due to an increased susceptibility to lymphoreticular disease such as leukaemia and lymphoma (G umyPause et al, 2004). ATM mutations have also been linked to breast cancer (Angele et al, 2003; Thorstenson et al, 2003). Thus, understanding the molecular basis for AT will yield important biological insights linking a diverse group of pathologies such as neurodegeneration, immune deficiency and cancer.

\section{Dysfunction of the ATM kinase is responsible for AT}

A major breakthrough in understanding AT came with the identification of a single gene, ATM (ataxia telangiectasia, mutated), which when mutated is the underlying cause of the disease (Savitsky et al, 1995). Indeed, as such a broad spectrum of organs are affected in AT, the discovery of ATM was hailed as a medical equivalent of the Rosetta stone (N owak, 1995). The identification of ATM has facilitated rapid progress in understanding many aspects of the molecular basis of this disease.

ATM has sequence homology to a family of proteins that are related to the phosphatidylinositol-3-O H-kinases (PI(3)K), although ATM is a protein kinase rather than a lipid kinase (Fig 2). ATM is a large protein; the genomic DNA contains 66 exons resulting in an mRNA of approximately $12 \mathrm{~kb}$ that encodes a protein of approximately $350 \mathrm{kDa}$. Mutations identified in ATM occur throughout the gene with no 'hot spots' and generally lead to protein instability (Lakin et al, 1996; Sandoval et al, 1999). Some mutations result in the production of decreased amounts of functional protein, or normal amounts with markedly reduced kinase activity. These mutations cause a milder version of AT with a less severe clinical phenotype, although neurodegeneration is still present (Stewart et al, 2001). A detailed ATM mutation database can be found at http://www.vmresearch.org/bri_investigators/atm.htm.

\section{ATM is a protein kinase that responds to DNA damage}

ATM is the apex of a signalling cascade that responds to DNA double-stranded breaks (DSBs) and is key to coordinating the resulting cellular response (Shiloh, 2003). It is also required for processing the physiological DNA strand breaks that occur during meiosis, immune system maturation and for telomere maintenance. ATM is a serine-threonine protein kinase that undergoes autophosphorylation after DNA damage to subsequently initiate a signalling cascade that involves the phosphorylation of several substrates (Kastan \& Lim, 2000; Shiloh, 2003). M any ATM substrates are cell-cycle regulators that have essential functions in the cellular response to DNA damage and include p53, breast-cancer-associated 1 (BRCA1), p53-binding protein 1 (53BP1) and the checkpoint kinase $\mathrm{CHK} 2$. The response to DNA damage appears to be the primary, if not the definitive, function of this kinase.

Recently, substantial insight has been achieved regarding the mechanism by which ATM signals that DNA has been damaged (Fig 3). In response to DSBs, ATM is autophosphorylated at Ser-1981, which leads to the dissociation of inactive multimeric ATM (either a dimer or higher order multimer) to initiate ATM signalling (Bakkenist \& Kastan, 2003). Although ATM is essential for the DSB response, it

\begin{abstract}
ATM
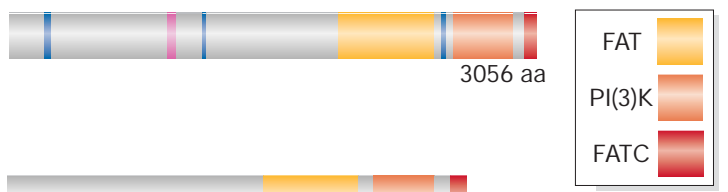

ATR

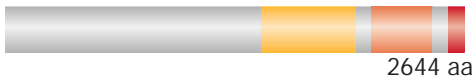

DNAPK $_{\text {CS }}$

2644 aa

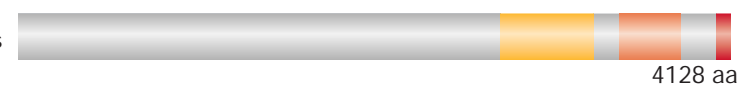

Fig 2 Thestructure of ATM . Ataxia telangiectasia, mutated (ATM) is a large protein that has carboxy-terminal similarity to other proteins of the phosphatidylinositol-3-OH-kinase( $\mathrm{PI}(3) \mathrm{K}$ ) family. Two other representative protein kinases-ATM - Rad3 related (ATR) and the catalytic subunit of the DNA-dependent protein kinase $\left(\right.$ DNAPK $_{\mathrm{cs}}$ ) - areincluded for comparison. Each of these PI (3)K members contain similar domains: a FAT domain, so named because it is a common motif in other related proteins, including FRAPP/ATM/TRRAP; thePI(3)K domain that was initially used to assign ATM to thePI(3)K family; and a FATC domain that represents a common C-terminal amino-acid sequence found near thetermini of the protein. The blue lines indicatenuclear localization signals and the pink lineindicates a leucinezipper region within ATM .
\end{abstract}

functions in concert with other factors. Foremost among these is the M RN complex (Carson et al, 2003; U ziel et al, 2003), named as such because of its three principal component proteins: M RE11, RAD 50 and NBS1 (D'Amours \& Jackson, 2002; Petrini \& Stracker, 2003; van den Bosch et al, 2003). Activated ATM can directly associate with the MRN complex, and this interaction can control signalling by influencing the ATM substrate choice (Lee \& Paull, 2004). Indeed, the important inter-relationship between ATM and the MRN complex is underscored by the similarity of two other syndromes related to AT that result from hypomorphic mutations in N BS1 and M RE11; $\mathrm{Nijmegen} \mathrm{breakage} \mathrm{syndrome} \mathrm{and} \mathrm{AT-like} \mathrm{disorder} \mathrm{(discussed} \mathrm{later).}$

In addition to ATM and MRN, other key players in the DSB response include histone $\mathrm{H} 2 \mathrm{AX}, 53 \mathrm{BP} 1$, mediator of damage checkpoint 1 (MDC1) and BRCA1. These factors are all substrates of ATM . After DNA damage, these factors rapidly mobilize to the sites of DSBs and initiate an ATM-dependent signalling cascade that leads to the resolution of the break through DNA repair, or, in the case of excessive DNA damage, cell death, often through p53-mediated apoptosis. Collectively, these proteins function as key regulators of the DNA damage response, and a clear interdependency exists among them as inactivation of any of them renders cells hypersensitive to DSBs (Kitagawa et al, 2004; Petrini \& Stracker, 2003; Sedelnikova et al, 2003; Shiloh, 2003; van den Bosch et al, 2003). Thus, ATM signalling after DSBs involves a coordinated series of events that occur rapidly and collectively serve to activate key cellular effectors (Shiloh, 2003).

\section{ATM controls cell-cycle checkpoints}

A crucial survival function when DSBs occur is the inhibition of the cell cycle through the activation of cell-cycle checkpoints. Checkpoints occur to introduce a pause in proliferation to address cellular stress. Although checkpoints can be easily demonstrated in cell-culture systems, the occurrence and role of these in vivo are less clear. However, the proteins that influence checkpoints are often required to prevent cancer. 
Factors involved in D NA damage responses are intimately linked to the activation of checkpoints. Because many ATM substrates are key effectors of the cell cycle, cells derived from AT individuals have defective cell-cycle checkpoints. For example, p53 is required for the $\mathrm{G} 1$ and $\mathrm{CHK} 2$ for the G2 DNA damage-induced checkpoints, whereas proteins such as BRCA1 and N BSI control the intra-S phase checkpoint. Therefore, the defective cell-cycle checkpoints present in AT cells after DNA damage represent the defective phosphorylation of ATM substrates (M otoyama \& Naka, 2004; Shiloh, 2003).

\section{ATM and cancer}

Cancer is linked to genomic instability and, consequently, many individuals suffering from syndromes that are characterized by defects in DNA damage responses are also cancer prone (Hoeijmakers, 2001; van $\mathrm{Gent}$ et al, 2001). Cancer occurs in about $10 \%$ of AT individuals and reflects the central role of ATM in the response to DSBs. However, despite the nervous system being markedly affected in AT, the tumour types occurring in this disease are primarily lymphoma or leukaemia (Gumy-Pause et al, 2004). Typical cytogenetic changes seen in tumours from AT individuals often involve aberrant oncogenic rearrangements at the T-cell receptor loci. The occurrence of these tumours underscores the requirement for ATM to ensure high-fidelity immunoglobulin-gene recombination after the normal DNA breakage and processing that occurs during immune system maturation (Liao \& Van Dyke, 1999; Perkins et al, 2002).

Somatic mutations in ATM have been identified in some sporadic cancers, particularly leukaemias (Boultwood, 2001; Stankovi et al, 2002; Thorstenson et al, 2003). Additionally, a substantial body of work has linked ATM heterozygotes to cancer predisposition (Angele et al, 2003; FitzGerald et al, 1997; Thorstenson et al, 2003). Recently, it has been shown that the nature of the particular ATM mutation has a substantial bearing on ATM heterozygote cancer susceptibility, as some mutant versions of ATM can act in a dominant interfering manner to partially disruptATM signalling (Spring et al, 2002).

\section{ATM, neurodegeneration and D NA damage}

The hallmark of AT is neurodegeneration. Understanding how dysfunctional ATM has an impact on the nervous system will first involve understanding the ATM signalling pathway in the brain and the aetiological agent that underlies the neurodegeneration. A clear picture of ATM function in the nervous system has yet to emerge, although substantial evidence supports a causal role in responding to DNA damage. Many human syndromes associated with DNA repair deficiencies also show neurological defects (Caldecott, 2003; Rolig \& McKinnon, 2000), which indicates that proper DNA damage responses are crucial for homeostasis of the nervous system.

Genetic insight into the requirement for DNA repair during nervous system development was initially obtained in mice in which the DNA ligase IV (Lig4) or the X-ray repair cross-complementing protein (XRCC4) had been deleted (Barnes et al, 1998; Gao et al, 1998). Inactivation of either of these partner proteins leads to midgestational embryonic lethality and is associated with abundant apoptosis throughout the entire neuraxis. Inactivation of other DNA repair genes have further established the broad requirement for DNA damage responses during development (Abner \& M cKinnon, 2004).

The early initiating events that involve ATM activation are likely to be similar in the nervous system to those described in vitro. However, in the nervous system, ATM response to DNA damage shows some important context dependency, and it is likely that the specific types
A
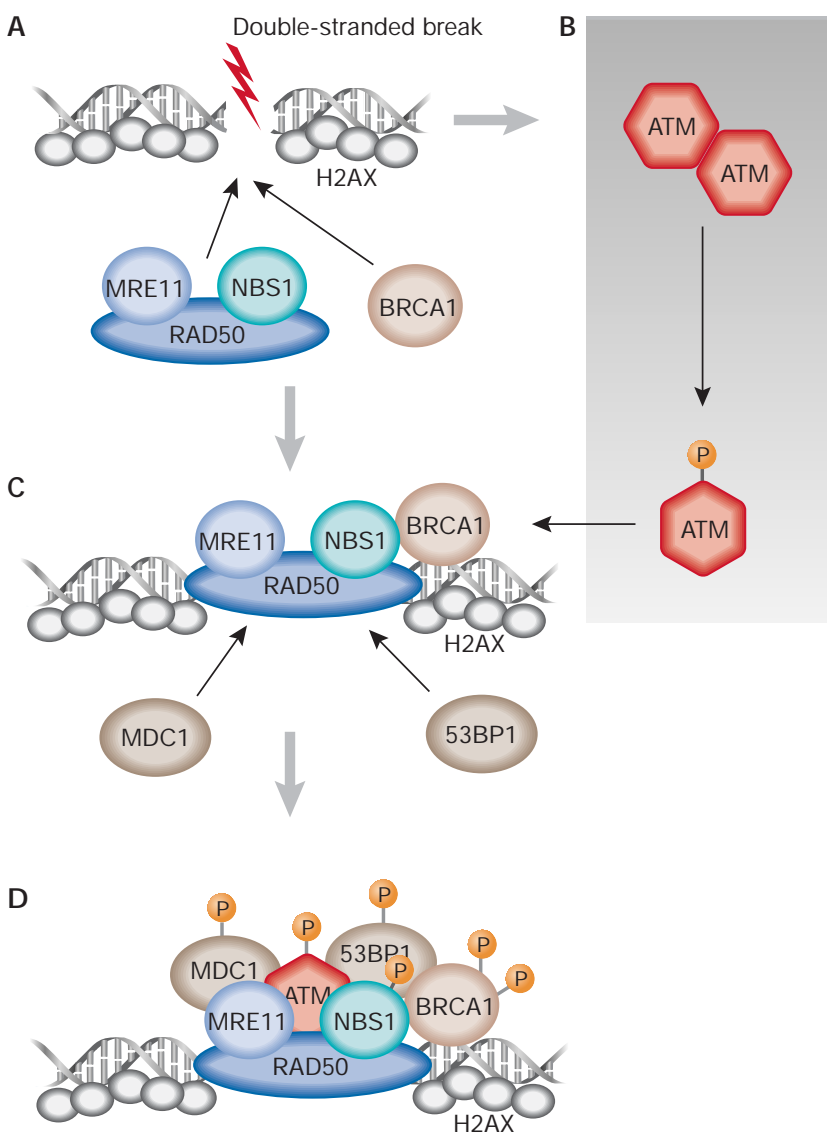

Fig3|ATM and DNA damagesignalling. In responseto a DNA doublestranded break (A) several simultaneous events occur to ultimately activate ATM signal transduction. ATM exists as an inactive multimeric complex that, in response to DNA damage, undergoes autophosphorylation to an active monomer (B). A histone variant, histone H2AX, present within chromatin, becomes phosphorylated and serves as a tethering platform for repair factors. The M RE11-RAD50-NBS1 complex locates to the DNA lesion together with BRCA1 (C). Assembly of this complex facilitates coordinated co-localization of active ATM together with other factorsincluding M DC1/NFBD 1 and 53BP1. BRCA 1, M DC 1 and 53BP1 arealso phosphorylated in an ATM dependent manner (D). The assembly of this multiprotein complex facilitates the cellular response to a DNA double-stranded break. 53BP1, p53-binding protein 1; ATM , ataxia telangiectasia, mutated; BRCA1, breast-cancerassociated 1; MDC1, mediator of damage checkpoint 1.

of DNA damage, and the nature of the cell type incurring this damage, are important determinants of ATM signal transduction (Borges et al, 2004; Herzog et al, 1998; Lee et al, 2001). ATM has been implicated directly in the response to endogenous damage in the nervous system as DNA lesions arising from Lig4 deficiency activate ATM to initiate neural apoptosis, and in Lig4/Atm double-null mice, apoptosis is abrogated (Lee et al, 2000; Sekiguchi et al, 2001). O ne function of ATM in the nervous system, therefore, is to eliminate neural cells that incur D N A damage, and failure to do this might lead to the accumulation of genetic lesions that eventually compromise cellular function and viability, causing cell death. Thus, DNA damage responses that engage ATM signalling are important in ensuring that genotoxic stress is relieved during neural development. 


\section{Ataxia-telangiectasia-related disorders}

Of particular relevance to DNA damage and AT are certain hypomorphic mutations of M RE11 that lead to a similar disease called ATLD. These individuals are also characterized by neurodegeneration, albeit less severe than AT (Stewart et al, 1999). In addition, mutations in NBS1, an ATM substrate that is involved in the DNA damage-induced intra-S phase checkpoint, lead to the Nijmegen breakage syndrome (NBS), which is a disease phenotypically similar to AT but with distinct neurological defects (Carney et al, 1998; Shiloh, 1997; Varon et al, 1998). In NBS, microcephaly is the neurological hallmark, rather than the progressive neurodegeneration that is seen in AT. The different ATLD and NBS neural phenotypes suggest some differential requirements for N BSI and M RE11 function in the nervous system. It is unclear how the principal signalling pathways that involve this multiprotein complex might function differently in nervous system development than in other tissues; the microcephaly characteristic of NBS compared with the neurodegeneration of ATLD suggest that MRN-ATM function is subject to other regulatory mechanisms in the nervous system. Recently, in vitro biochemical analyses have suggested independent functions for the MRN complex and a complex of only MRE11 and RAD50 (MR) that involve the selective activation of different ATM substrates. In this scenario, ATM activation by the MR complex activates p53, whereas the binding of ATM to the MRN complex activates CHK2 (Lee \& Paull, 2004).

The case for DNA damage as a primary factor in AT-associated neurodegeneration is strong, but increased oxidative stress resulting from ATM deficiency in the nervous system has been reported, although the mechanism for this feature is unclear (Barlow et al, 1999; Kamsler et al, 2001; Q uick \& D ugan, 2001). Whether this is a primary or secondary effect of ATM deficiency is also not known.

\section{Conclusions}

Is D N A damage a common denominator for the AT phenotype? The simplest interpretation for the role of ATM in preventing AT is that it ensures an appropriate response to DNA damage. This aspect of ATM function explains the immune-system defects that require gene rearrangements for immune maturation, and also the development of lymphoma or leukaemia. Radiosensitivity is also clearly linked to a defective DNA damage response, and sterility results from defects early in meiosis that involve genetic recombination events (Barlow et al, 1997). However, some features, such as ocular telangiectasia and insulin resistance, are more difficult to reconcile with a defective D NA damage response.

It has been most difficult to assign a molecular basis to neurodegeneration. This is largely because of the relative difficulty of working with neural tissues and neuronal cultures compared with standard culture approaches using transformed cells. However, defective DNA damage responses underlie the molecular basis of other neurodegenerative syndromes that are characterized by ataxia similar to AT. In fact, the most common recessive ataxia in Japan and the second most common in Portugal (after Friedrich's ataxia) - ataxiaocular apraxia 1- has very similar neuropathology to AT. It results from mutations in aprataxin, which is a protein that is involved in the response to single-stranded breaks in DNA rather than DSBs (D ate et al, 2001; Gueven et al, 2004; Moreira et al, 2001). It is likely that an increasing number of uncharacterized ataxias and neurological diseases will be shown to be the result of inappropriate responses to DNA damage.
Whereas a great deal of insight has come from studies with AT cells, there is still much that is unresolved about ATM function. An important undertaking will be to integrate the biochemical signalling involving the numerous substrates of ATM into a cohesive biological picture that accounts for the pleiotropic AT phenotype. In particular, it will be important to understand the molecular basis of the tissuespecific functions of ATM in responding to DNA damage (Baker \& McKinnon, 2004). For example, why are some tissues, such as the haematopoietic and intestinal tract, hypersensitive to DNA-damageinduced cell death when ATM is dysfunctional, whereas other organs, such as the developing brain, are resistant? How does the resistance of immature neural cells to DNA-damage-induced cell death relate to neurodegeneration; do these cells subsequently die from accumulated genetic lesions? Understanding tissue-specific ATM function will provide important insights into context-dependent consequences of DNA damage that will have broad biological implications.

\section{ACKN O W LED GEMENTS}

I thank S. Baker for discussion and comments on themanuscript. Space constraints limited citations to reviews and recent publications. Support for this work was from the National Institutes of $\mathrm{H}$ ealth and theAmerican Lebanese and Syrian Associated Charities of St JudeChildren's Research H ospital.

\section{REFEREN CES}

Abner CW, M cKinnon PJ (2004)The DNA double-strand break response in the nervous system. DNA Repair (Amst) (in press)

Angele S et al (2003) ATM haplotypes and cellular response to DNA damage: association with breast cancer risk and clinical radiosensitivity. Cancer Res 63: 8717-8725

Baker SJ, M cKinnon PJ (2004)Tumour-suppressor function in the nervous system. N at Rev Cancer 4: 184-196

Bakkenist CJ, Kastan M B (2003) DN A damage activates ATM through intermolecular autophosphorylation and dimer dissociation. Nature 421: 499-506

Barlow C, Liyanage M, M oens PB, Deng CX, Ried T, Wynshaw-Boris A (1997) Partial rescue of the prophase I defects of Atm-deficient mice by $\mathrm{p} 53$ and p21 null alleles. $N$ at $\mathrm{G}$ enet 17: 462-466

Barlow C, Dennery PA, Shigenaga M K, Smith M A, M orrow JD, Roberts LI II, Wynshaw-Boris A, Levine RL (1999) Loss of the ataxia-telangiectasia gene product causes oxidative damage in target organs. Proc $\mathrm{N}$ atl Acad Sci USA 96: 9915-9919

Barnes DE, Stamp G, Rosew ell I, Denzel A, Lindahl T (1998)Targeted disruption of the gene encoding D N A ligase IV leads to lethality in embryonic mice. Curr Biol 8: 1395-1398

Borges HL, Chao C, XuY, Linden R, Wang JY (2004) Radiation-induced apoptosis in developing mouse retina exhibits dose-dependent requirement for ATM phosphorylation of p53. Cell Death Differ 11: 494-502

Boultwood J (2001) A taxia telangiectasia gene mutations in leukaemia and Iymphoma. I Clin Pathol 54: 512-516

Caldecott KW (2003) DNA single-strand break repair and spinocerebellar ataxia. Cell 112: 7-10

Carney JP, Maser RS, O livares H, D avis EM, Le Beau M, Yates IR III, H ays L, M organ W F, Petrini JH (1998)The hM rel1/hRad50 protein complex and $\mathrm{Nijmegen} \mathrm{breakage} \mathrm{syndrome:} \mathrm{linkage} \mathrm{of} \mathrm{double-strand} \mathrm{break} \mathrm{repair} \mathrm{to}$ the cellular DNA damage response. Cell 93: 477-486

Carson CT, Schwartz RA, StrackerTH, Lilley CE, Lee DV, Weitzman M D (2003) The M rell complex is required for ATM activation and the $G(2) / M$ checkpoint. EM BO 」 22: 6610-6620

Crawford TO (1998) Ataxia telangiectasia. Semin Pediatr N eurol 5: 287-294

D'Amours D, Jackson SP (2002) The M rell complex: at the crossroads of DNA repair and checkpoint signalling. Nat Rev Mol Cell Biol 3: 317-327

Date $\mathrm{H}$ et al(2001) Early-onset ataxia with ocular motor apraxia and hypoalbuminemia is caused by mutations in a new HIT superfamily gene. Nat Genet 29: 184-188

Farina L, U ggetti C, O ttolini A, M artelli A, Bergamaschi R, Sibilla L, Zappoli F, Egitto M G, Lanzi G (1994) Ataxia-telangiectasia: M R and CT findings. J Comput Assist Tomogr 18: 724-727 
FitzG erald MG, Bean JM, H egde SR, Unsal H, M acD onald DJ, H arkin DP, Finkelstein D M, Isselbacher KJ, H aber DA (1997) H eterozygous ATM mutations do not contribute to early onset of breast cancer. N at G enet 15 : 307-310

Gao Y et al (1998) A critical role for DNA end-joining proteins in both lymphogenesis and neurogenesis. Cell 95: 891-902

$\mathrm{G}$ atti RA et al (2001) The pathogenesis of ataxia-telangiectasia. Learning from a Rosetta stone. Clin Rev Allergy Immunol 20: 87-108

Gueven N et al (2004) Aprataxin, a novel protein that protects against genotoxic stress. Hum Mol Genet 13: 1081-1093

Gumy-Pause F, Wacker P, Sappino AP (2004) ATM gene and lymphoid malignancies. Leukemia 18: 238-242

Herzog KH, Chong MJ, Kapsetaki M , M organ JI, M cKinnon PJ (1998) Requirement for Atm in ionizing radiation-induced cell death in the developing central nervous system. Science 280: 1089-1091

Hoeijmakers JH (2001) Genome maintenance mechanisms for preventing cancer. N ature 411: 366-374

Kamsler A, Daily D, Hochman A, Stern N, Shiloh Y, Rotman G, Barzilai A (2001) Increased oxidative stress in ataxia telangiectasia evidenced by alterations in redox state of brains from Atm-deficient mice. Cancer Res 61: $1849-1854$

Kastan M B, Lim DS (2000) The many substrates and functions of ATM . N at Rev Mol Cell Biol 1: 179-186

Kitagawa R, Bakkenist CJ, M cKinnon PJ, Kastan M B (2004) Phosphorylation of SM C1 is a critical downstream event in the ATM-N BS1-BRCA1 pathway. Genes Dev 18: 1423-1438

Lakin N D, W eber P, Stankovic T, Rottinghaus ST, Taylor AM, Jackson SP (1996) Analysis of the ATM protein in wild-type and ataxia telangiectasia cells. Oncogene 13: 2707-2716

Lee JH, Paull TT (2004) Direct activation of the ATM protein kinase by the M re11/Rad50/N bs1 complex. Science 304: 93-96

LeeY, Barnes DE, LindahI T, M CKinnon PJ (2000) D efective neurogenesis resulting from $D$ NA ligase IV deficiency requires $A$ tm. G enes D ev 14: 2576-2580

LeeY, Chong MJ, M cKinnon PJ (2001) Ataxia telangiectasia mutateddependent apoptosis after genotoxic stress in the developing nervous system is determined by cellular differentiation status. J N eurosci $\mathbf{2 1}$ 6687-6693

Lefton-Greif M A, Crawford TO, W inkelstein JA, Loughlin GM, Koerner CB, Zahurak M, Lederman HM (2000) O ropharyngeal dysphagia and aspiration in patients with ataxia-telangiectasia. J Pediatr 136: 225-231

Lewis RF, Lederman HM , Crawford TO (1999) O cular motor abnormalities in ataxia telangiectasia. Ann N eurol 46: 287-295

Liao MJ, Van DykeT (1999) Critical role for Atm in suppressing V(D)] recombination-driven thymic lymphoma. Genes D ev 13: 1246-1250

Moreira MC et al (2001) The gene mutated in ataxia-ocular apraxia 1 encodes the new HIT/Zn-finger protein aprataxin. N at Genet 29: 189-193

M otoyama N, Naka K (2004) D N A damage tumor suppressor genes and genomic instability. Curr O pin Genet Dev 14: 11-16

N owak R (1995) Discovery of AT gene sparks biomedical research bonanza. Science 268: 1700-1701

N owak-W egrzyn A, Crawford TO, Winkelstein JA, Carson KA, Lederman H M (2004) Immunodeficiency and infections in ataxia-telangiectasia. J Pediatr 144: 505-511
Perkins EJ, N air A, Cowley D O, Van DykeT, Chang Y, Ramsden DA (2002) Sensing of intermediates in V(D)] recombination by ATM. G enes D ev 16: 159-164

Perlman S, Becker-Catania S, Gatti RA (2003) Ataxia-telangiectasia: diagnosis and treatment. Semin Pediatr N eurol 10: 173-182

Petrini JH, StrackerTH (2003)The cellular response to DNA double-strand breaks: defining the sensors and mediators. Trends Cell Biol 13: 458-462

Q uick KL, Dugan LL (2001) Superoxide stress identifies neurons at risk in a model of ataxia-telangiectasia. Ann N eurol 49: 627-635

Rolig RL, M CKinnon PJ (2000) Linking D N A damage and neurodegeneration. Trends N eurosci 23: 417-424

Sandoval N et al (1999) Characterization of ATM gene mutations in 66 ataxia telangiectasia families. H um Mol Genet 8: 69-79

Savitsky K et al (1995) A single ataxia telangiectasia gene with a product similar to PI-3 kinase. Science 268: 1749-1753

Sedelnikova OA, Pilch DR, Redon C, Bonner W M (2003) Histone H2AX in DNA damage and repair. Cancer Biol Ther 2: 233-235

Sedgwick RP, Boder E (1991) Ataxia-telangiectasia. In Handbook of Clinical Neurology Vol 60 (eds Vinken P, Bruyn G, Klawans H) 347-423. N ew York, USA: Elsevier.

Sekiguchi J et al (2001) Genetic interactions between ATM and the nonhomologous end-joining factors in genomic stability and development. Proc N atl Acad Sci U SA 98: 3243-3248

Shiloh Y (1997) A taxia-telangiectasia and the Nijmegen breakage syndrome: related disorders but genes apart. Annu Rev G enet 31: 635-662

ShilohY (2003) ATM and related protein kinases: safeguarding genome integrity. N at Rev Cancer 3: 155-168

Spring K et al (2002) M ice heterozygous for mutation in Atm, the gene involved in ataxia-telangiectasia, have heightened susceptibility to cancer. N at Genet 32: 185-190

Stankovi T, Stewart GS, Byrd P, Fegan C, M oss PA, TaylorAM (2002) ATM mutations in sporadic lymphoid tumours. Leuk Lymphoma 43: 1563-1571

Stewart GS et al (1999) The DNA double-strand break repair gene hM RE11 is mutated in individuals with an ataxia-telangiectasia-like disorder. Cell 99: 577-587

Stewart GS, LastJI, Stankovic T, H aites N, Kidd AM , Byrd PJ, Taylor AM (2001) Residual ataxia telangiectasia mutated protein function in cells from ataxia telangiectasia patients, with 5762 ins 137 and $7271 \mathrm{~T} \rightarrow \mathrm{G}$ mutations, showing a less severe phenotype. J Biol Chem 276: 30133-30141

Tavani F, Zimmerman RA, Berry GT, Sullivan K, G atti R, Bingham P (2003) Ataxia-telangiectasia: the pattern of cerebellar atrophy on $\mathrm{MRI}$. Neuroradiology 45: 315-319

Thorstenson YR et al (2003) Contributions of ATM mutations to familial breast and ovarian cancer. Cancer Res 63: 3325-3333

U ziel T, Lerenthal Y, M oyal L, Andegeko Y, M ittelman L, ShilohY (2003) Requirement of the MRN complex for ATM activation by DNA damage. EM BO J 22: 5612-5621

van den Bosch M, Bree RT, Lowndes NF (2003) The M RN complex: coordinating and mediating the response to broken chromosomes. EM BO Rep 4: 844-849

van Gent DC, Hoeijmakers JH, Kanaar R (2001) Chromosomal stability and the DNA double-stranded break connection. N at Rev Genet 2: 196-206

Varon R et al (1998) N ibrin, a novel DNA double-strand break repair protein, is mutated in Nijmegen breakage syndrome. Cell 93: 467-476 